\title{
Wakes in dilatonic current-carrying cosmic strings
}

\author{
A. L. N. Oliveira* \\ Instituto de Física, Universidade de Brasília, Brasília, Brazil \\ M. E. X. Guimarães \\ Instituto de Física Teórica/UNESP, São Paulo, Brazil \\ (Received 12 March 2003; published 20 June 2003)
}

\begin{abstract}
In this work, we present the gravitational field generated by a cosmic string carrying a timelike current in the scalar-tensor gravities. The mechanism of formation and evolution of wakes is fully investigated in this framework. We show explicitly that the inclusion of electromagnetic properties for the string induces logarithmic divergences in the accretion problem.
\end{abstract}

DOI: 10.1103/PhysRevD.67.123514

PACS number(s): $98.80 . \mathrm{Cq}$

\section{INTRODUCTION}

Topological defects are predicted in many gauge models as solitonic solutions resulting from spontaneous breaking of gauge or global symmetries. Among all these solutions, cosmic strings have attracted attention because they may be the source of large-scale structure in the Universe [1]. A relevant mechanism to understand structure formation by cosmic strings involves long strings moving with relativistic speed in the normal plane, giving rise to velocity perturbations in their wake [2]. The mechanism of forming wakes has been considered by many authors in the general relativity theory $[3,4]$. More recently, Masalskiene and Guimarães [5] considered the formation of wakes by long strings in the context of scalar-tensor theories. They showed that the presence of a gravitational scalar field-which from now on we will call generically a "dilaton"-induces a very similar structure as in the case of a wiggly cosmic string [4]. Further, Bezerra and Ferreira [6] showed that, if torsion is present, this effect is amplified.

Our purpose in this paper is twofold. We first generalize the work of Peter and Puy [7] and of Ferreira et al. [8] for the case of a cosmic string carrying a timelike current in the framework of scalar-tensor gravities. From the point of view of purely gravitational physics, it was shown that the inclusion of a current hardly affects the metric outside the string [9]. However, it is well known that the inclusion of such an internal structure can change the predictions of cosmic string models in the microwave background anisotropies $[10,11]$. This is our second goal; namely, we analyze the formation and evolution of wakes in this spacetime and we show explicitly how the current affects this mechanism. For this purpose, we consider a model in which nonbaryonic cold dark matter propagates around the current-carrying string. The Zel'dovich approximation is carried out in order to treat this motion. We anticipate that our main result is to show that the inclusion of a current brings logarithmic divergences and can actually break down the accretion mechanism by wakes.

This work is outlined as follows. In Sec. II, after setting

\footnotetext{
*Email address: andreo@fis.unb.br

†Email address: emilia@ift.unesp.br
}

the relevant microscopic model which describes a superconducting string carrying a current of timelike type, we present its gravitational field and show the explicit dependence of the deficit angle, the deflection of light, and the geodesics on the timelike current. In Sec. III, we consider the mechanism of formation and evolution of wakes in this framework by means of the Zel'dovich approximation. Finally, in Sec. IV, we end up with some conclusions and remarks.

\section{TIMELIKE CURRENT-CARRYING STRINGS}

In this section we study the gravitational field generated by a string carrying a current of timelike type. We start with the action in the Jordan-Fierz frame

$$
\begin{aligned}
\mathcal{S}= & \frac{1}{16 \pi} \int d^{4} x \sqrt{-\widetilde{g}}\left(\widetilde{\Phi} \widetilde{R}-\frac{\omega(\widetilde{\Phi})}{\widetilde{\Phi}} \tilde{g}^{\mu \nu} \partial_{\mu} \widetilde{\Phi} \partial_{\nu} \widetilde{\Phi}\right) \\
& +\mathcal{S}_{m}\left[\psi_{m}, \tilde{g}_{\mu \nu}\right] .
\end{aligned}
$$

$\tilde{g}_{\mu \nu}$ is the physical metric which contains both scalar and tensor degrees of freedom, $\widetilde{R}$ is the curvature scalar associated with it, and $\mathcal{S}_{m}$ is the action for general matter fields which, at this point, is left arbitrary. The metric signature is assumed to be $(-,+,+,+)$.

In what follows, we concentrate our attention on superconducting vortex configurations which arise from the spontaneous breaking of the symmetry $U(1) \times U_{e m}(1)$. Therefore, the action for the matter fields is composed of two pairs of coupled complex scalar and gauge fields $\left(\varphi, B_{\mu}\right)$ and $\left(\sigma, A_{\mu}\right)$. Also, for technical purposes, it is preferable to work in the so-called Einstein (or conformal) frame, in which the scalar and tensor degrees of freedom do not mix:

$$
\begin{aligned}
\mathcal{S}= & \frac{1}{16 \pi G_{*}} \int d^{4} x \sqrt{-g}\left[R-2 \partial_{\mu} \phi \partial^{\mu} \phi\right]+\int d^{4} x \sqrt{-g} \\
& \times\left(-\frac{1}{2} \Omega^{2}(\phi)\left[\left(D_{\mu} \varphi\right)^{*} D^{\mu} \varphi+\left(D_{\mu} \sigma\right)^{*} D^{\mu} \sigma\right]\right. \\
& \left.-\frac{1}{16 \pi}\left(F_{\mu \nu} F^{\mu \nu}+H_{\mu \nu} H^{\mu \nu}\right)-\Omega^{2}(\phi) V(|\varphi|,|\sigma|)\right),
\end{aligned}
$$


where $F_{\mu \nu}=\partial \mu A_{\nu}-\partial_{\nu} A_{\mu}, H_{\mu \nu}=\partial \mu B_{\nu}-\partial_{\nu} B_{\mu}$, and the potential is suitably chosen in order that the pair $\left(\varphi, B_{\mu}\right)$ breaks one symmetry $U(1)$ in vacuum (giving rise to the vortex configuration) and the second pair $\left(\sigma, A_{\mu}\right)$ breaks the symmetry $U_{e m}(1)$ in the core of the vortex (giving rise to the superconducting properties):

$$
\begin{aligned}
V(|\varphi|,|\sigma|)= & \frac{\lambda_{\varphi}}{8}\left(|\varphi|^{2}-\eta^{2}\right)^{2}+f\left(|\varphi|^{2}-\eta^{2}\right)|\sigma|^{2}+\frac{\lambda_{\sigma}}{4}|\sigma|^{4} \\
& +\frac{m_{\sigma}^{2}}{2}|\sigma|^{2}
\end{aligned}
$$

We restrict ourselves, then, to the configurations corresponding to an isolated, static current-carrying vortex lying on the $z$ axis. In a cylindrical coordinate system $(t, r, z, \theta)$ such that $r \geqslant 0$ and $0 \leqslant \theta<2 \pi$, we make the following ansatz:

$$
\varphi=\varphi(r) e^{i \theta}, \quad B_{\mu}=\frac{1}{q}[Q(r)-1] .
$$

The pair $\left(\sigma, A_{\mu}\right)$, which is responsible for the superconducting properties of the vortex, is set in the form

$$
\sigma=\sigma(r) e^{i \chi(t)}, \quad A_{t}=\frac{1}{e}\left[P_{t}(r)-\partial_{t} \chi\right],
$$

where $P_{t}$ corresponds to the electric field that leads to a timelike current in the vortex. We also require that the functions $\varphi, Q(r), \sigma(r)$, and $P_{t}$ must be regular everywhere and must satisfy the usual boundary conditions of vortex [12] and superconducting configurations $[13,14]$.

The action (2) is obtained from Eq. (1) by a conformal transformation

$$
\tilde{g}_{\mu \nu}=\Omega^{2}(\phi) g_{\mu \nu}
$$

and by the redefinition of the quantity

$$
G_{*} \Omega^{2}(\phi)=\widetilde{\Phi}^{-1},
$$

which makes evident the feature that any gravitational phenomena will be affected by the variation of the gravitation constant $G_{*}$ in the scalar-tensor gravity, and by introducing a new parameter

$$
\alpha^{2}=\left(\frac{\partial \ln \Omega(\phi)}{\partial \phi}\right)^{2}=[2 \omega(\widetilde{\Phi})+3]^{-1} .
$$

Variation of the action (2) with respect to the metric $g_{\mu \nu}$ and to the dilaton field $\phi$ gives the modified Einstein's equations and a wave equation for the dilaton, respectively; namely,

$$
\begin{aligned}
G_{\mu \nu} & =2 \partial_{\mu} \phi \partial_{\nu} \phi-g_{\mu \nu}{ }^{\alpha \beta} \partial_{\alpha} \phi \partial_{\beta} \phi+8 \pi G_{*} T_{\mu \nu}, \\
\square_{g} \phi & =-4 \pi G_{*} \alpha(\phi) T,
\end{aligned}
$$

where $T_{\mu \nu}$ is the energy-momentum tensor, which is obtained by

$$
T_{\mu \nu}=\frac{-2}{\sqrt{-g}} \frac{\delta S_{m a t}}{\delta g_{\mu \nu}} .
$$

We note in passing that, in the conformal frame, this tensor is not conserved, providing us with an additional equation

$$
\nabla_{\mu} T_{\nu}^{\mu}=\alpha(\phi) T \nabla_{\nu} \phi
$$

In what follows, we will write the general static metric with cylindrical symmetry corresponding to the electric case in the form

$$
d s^{2}=-e^{2 \psi} d t^{2}+e^{2(\gamma-\psi)}\left(d r^{2}+d z^{2}\right)+\beta^{2} e^{-2 \psi} d \theta^{2},
$$

where $\psi, \gamma, \beta$ are functions of $r$ only.

The nonvanishing components of the energy-momentum tensor using Eq. (7) are

$$
\begin{aligned}
T_{t}^{t}= & -\frac{1}{2} \Omega^{2}(\phi)\left\{-g^{t t} \sigma^{2} P_{t}^{2}+g^{r r}\left[\varphi^{\prime 2}+\sigma^{\prime 2}\right]+g^{\theta \theta} \varphi^{2} Q^{2}\right\} \\
& -\frac{1}{8 \pi} g^{r r}\left\{-g^{t t} \frac{P_{t}^{\prime 2}}{e^{2}}+g^{\theta \theta} \frac{Q^{\prime 2}}{q^{2}}\right\}-\Omega^{4}(\phi) V(\sigma, \varphi), \\
T_{r}^{r}= & -\frac{1}{2} \Omega^{2}(\phi)\left\{g^{t t} \sigma^{2} P_{t}^{2}-g^{r r}\left[\varphi^{\prime 2}+\sigma^{\prime 2}\right]+g^{\theta \theta} \varphi^{2} Q^{2}\right\} \\
& +\frac{1}{8 \pi} g^{r r}\left\{g^{t t} \frac{P_{t}^{\prime 2}}{e^{2}}+g^{\theta \theta} \frac{Q^{\prime 2}}{q^{2}}\right\}-\Omega^{4}(\phi) V(\sigma, \varphi), \\
T_{\theta}^{\theta}= & -\frac{1}{2} \Omega^{2}(\phi)\left\{g^{t t} \sigma^{2} P_{t}^{2}+g^{r r}\left[\varphi^{\prime 2}+\sigma^{\prime 2}\right]-g^{\theta \theta} \varphi^{2} Q^{2}\right\} \\
& -\frac{1}{8 \pi} g^{r r}\left\{g^{t t} \frac{P_{t}^{\prime 2}}{e^{2}}-g^{\theta \theta} \frac{Q^{\prime 2}}{q^{2}}\right\}-\Omega^{4}(\phi) V(\sigma, \varphi), \\
T_{z}^{z}= & -\frac{1}{2} \Omega^{2}(\phi)\left\{g^{t t} \sigma^{2} P_{t}^{2}+g^{r r}\left[\varphi^{\prime 2}+\sigma^{\prime 2}\right]+g^{\theta \theta} \varphi^{2} Q^{2}\right\} \\
& -\frac{1}{8 \pi} g^{r r}\left\{g^{t t} \frac{P_{t}^{\prime 2}}{e^{2}}+g^{\theta \theta} \frac{Q^{\prime 2}}{q^{2}}\right\}-\Omega^{4}(\phi) V(\sigma, \varphi) .
\end{aligned}
$$

Therefore, for the electric case, Eqs. (6) are written as

$$
\begin{aligned}
\beta^{\prime \prime} & =8 \pi G_{*} e^{2(\gamma-\psi)} \beta\left[T_{1}^{1}+T_{3}^{3}\right], \\
\left(\beta \psi^{\prime}\right)^{\prime} & =4 \pi G_{*} e^{2(\gamma-\psi)} \beta\left[-T_{0}^{0}+T_{1}^{1}+T_{2}^{2}+T_{3}^{3}\right], \\
\beta^{\prime} \gamma^{\prime} & =\beta\left(\psi^{\prime}\right)^{2}+\beta\left(\phi^{\prime}\right)^{2}+8 \pi G_{*} \beta e^{2(\gamma-\psi)} T_{1}^{1}, \\
\left(\beta \phi^{\prime}\right)^{\prime} & =-4 \pi G_{*} \beta e^{2(\gamma-\psi)} T .
\end{aligned}
$$

In order to solve the above equations we will divide the space into two regions: the exterior region $r \geqslant r_{0}$, in which only the electric component of the Maxwell tensor contributes to the energy-momentum tensor, and the internal region $0 \leqslant r<r_{0}$, where all matter fields survive. $r_{0}$ is the string thickness. 


\section{A. The exterior metric}

Due to the specific properties of the Maxwell tensor

$$
T_{\mu}^{\mu}=0 \quad \text { and } \quad T_{\nu}^{\alpha} T_{\alpha}^{\mu}=\frac{1}{4}\left(T^{\alpha \beta} T_{\alpha \beta}\right) \delta_{\nu}^{\mu}
$$

the Einstein equations (10) may be transformed into some algebraic relations called Rainich algebra $[15,16]$ which, for the electric case, have the form ${ }^{1}$

$$
\begin{gathered}
R=R_{z}^{z}+R_{r}^{r}+R_{\theta}^{\theta}+R_{t}^{t}=2 e^{2(\psi-\gamma)} \phi^{\prime 2}, \\
\left(R_{t}^{t}\right)^{2}=\left(R_{r}^{r}-2 g^{r r} \phi^{\prime 2}\right)^{2}=\left(R_{\theta}^{\theta}\right)^{2}=\left(R_{z}^{z}\right)^{2}, \\
R_{t}^{t}=-R_{\theta}^{\theta}, \quad R_{t}^{t}=R_{r}^{r}-2 g^{r r} \phi^{\prime 2}, \quad R_{\theta}^{\theta}=R_{z}^{z} .
\end{gathered}
$$

Then we have

$$
\begin{aligned}
\psi^{\prime \prime}+\frac{1}{r} \psi^{\prime}-\psi^{\prime 2}-\gamma^{\prime \prime} & =\frac{D^{2}}{r^{2}}, \\
\gamma^{\prime \prime}+\frac{1}{r} \gamma^{\prime} & =0 .
\end{aligned}
$$

The solution is

$$
\begin{aligned}
& \gamma=m^{2} \ln \left(\frac{r}{r_{0}}\right), \\
& \psi=n \ln \left(\frac{r}{r_{0}}\right)-\ln \left(\frac{r / r_{0}+k}{1+k}\right) .
\end{aligned}
$$

Therefore, the exterior metric for a timelike currentcarrying string is

$$
\begin{aligned}
d s_{E}^{2}= & \left(\frac{r}{r_{0}}\right)^{2 m^{2}-2 n} W^{2}(r)\left(d r^{2}+d z^{2}\right) \\
& +\left(\frac{r}{r_{0}}\right)^{-2 n} W^{2}(r) B^{2} r^{2} d \theta^{2}-\left(\frac{r}{r_{0}}\right)^{2 n} \frac{1}{W^{2}(r)} d t^{2},
\end{aligned}
$$

where

$$
W(r) \equiv \frac{\left(r / r_{0}\right)^{2 n}+k}{1+k}
$$

The constants $m, n, k, D$ will be determined after the inclusion of the matter fields.

\section{B. The internal metric and the matching}

In order to solve the Einstein equations (6) in the internal region (where all matter fields contribute to the energy-

\footnotetext{
${ }^{1}$ In scalar-tensor theories, these relations are modified by a term that depends on the dilaton [8].
}

momentum tensor) we use the weak-field approximation. Therefore, we can expand the metric and the dilaton fields to first order in $G_{0}$ such that

$$
\begin{gathered}
g_{\mu \nu}=\eta_{\mu \nu}+h_{\mu \nu}, \\
\phi=\phi_{0}+\phi_{1} .
\end{gathered}
$$

In this way, Eqs. (6) reduce to

$$
\nabla^{2} h_{\mu \nu}=-16 \pi G_{0}\left(T_{\mu \nu}^{(0)}-\frac{1}{2} \eta_{\mu \nu} T^{(0)}\right)
$$

in a harmonic coordinate system such that $\left(h_{\nu}^{\mu}-\frac{1}{2} \delta_{\nu}^{\mu} h\right)_{, \nu}$ $=0$, and the linearized equation for the dilaton field is

$$
\nabla^{2} \phi_{(1)}=-4 \pi G_{0} \alpha\left(\phi_{0}\right) T^{(0)}
$$

In the above equations (18) and (19), $T_{\mu \nu}^{(0)}$ is the energymomentum tensor evaluated to zeroth order in $G_{0}$, and $T^{(0)}$ its trace. In Cartesian (harmonic) coordinates the nonvanishing components of $T_{\mu \nu}^{(0)}$ are

$$
\begin{aligned}
T_{(0) t}^{t}= & -\frac{1}{2}\left\{\sigma^{2} P_{t}^{2}+\varphi^{\prime 2}+\sigma^{\prime 2}+\frac{1}{r^{2}} \varphi^{2} Q^{2}\right\} \\
& -\frac{1}{8 \pi}\left\{-\frac{P_{t}^{\prime 2}}{e^{2}}+\frac{1}{r^{2}} \frac{Q^{\prime 2}}{q^{2}}+\right\}-V(\sigma, \varphi),
\end{aligned}
$$

$$
T_{(0) x}^{x}=\left(\cos ^{2}(\theta)-\frac{1}{2}\right)\left\{\sigma^{\prime 2}+\varphi^{\prime 2}-\frac{\varphi^{2} Q^{2}}{r^{2}}-\frac{P_{t}^{\prime 2}}{4 \pi e^{2}}\right\}
$$$$
-\frac{1}{2}\left\{-\sigma^{2} P_{t}^{2}-\frac{1}{4 \pi} \frac{Q^{\prime}}{r^{2} q^{2}}+2 V(\sigma, \phi)\right\},
$$$$
T_{(0) y}^{y}=\left(\sin ^{2}(\theta)-\frac{1}{2}\right)\left\{\varphi^{\prime 2}+\sigma^{\prime 2}-\frac{\varphi^{2} Q^{2}}{r^{2}}-\frac{P_{t}^{\prime 2}}{e 4 \pi^{2}}\right\}
$$$$
-\frac{1}{2}\left\{-\sigma^{2} P_{t}^{2}-\frac{1}{4 \pi} \frac{Q^{\prime 2}}{r^{2} q^{2}}+2 V(\sigma, \phi)\right\},
$$

$$
\begin{aligned}
T_{(0) z}^{z}= & -\frac{1}{2}\left\{-\sigma^{2} P_{t}^{2}+\varphi^{\prime 2}+\sigma^{\prime 2}+\frac{1}{r^{2}} \varphi^{2} Q^{2}\right\} \\
& -\frac{1}{8 \pi}\left\{-\frac{P_{t}^{\prime 2}}{e^{2}}+\frac{1}{r^{2}} \frac{Q^{\prime 2}}{q^{2}}\right\}-V(\sigma, \varphi) .
\end{aligned}
$$

In this paper, in order to solve Eqs. (18) and (19) with source given by Eqs. (20), we use a method that was first applied by Linet in Ref. [17] and later by Peter and Puy [7] and by Ferreira et al. [8]. The trick consists in writing down the 
string and its component fields by means of $\delta$ functions. ${ }^{2}$ In doing this, we are allowed to write the string's energymomentum tensor as

$$
T^{\mu \nu}=\operatorname{diag}(U,-Z,-Z,-T),
$$

where $U, Z$, and $T$ are macroscopic quantities which are defined as the energy per unit length, the transverse components per unit length, and the tension per unit length, respectively:

$$
\begin{aligned}
& U \equiv M_{t}^{t}=-2 \pi \int_{0}^{r_{0}} T_{t}^{t} r d r, \\
& X \equiv M_{r}^{r}=-2 \pi \int_{0}^{r_{0}} T_{r}^{r} r d r \\
& Y \equiv M_{\theta}^{\theta}=-2 \pi \int_{0}^{r_{0}} T_{\theta}^{\theta} r d r, \\
& T \equiv M_{z}^{z}=-2 \pi \int_{0}^{r_{0}} T_{z}^{z} r d r .
\end{aligned}
$$

From the transverse quantities $X$ and $Y$ we define one single Cartesian component $Z$ :

$$
Z=-\int r d r d \theta T_{x}^{x}=-\int r d r d \theta T_{y}^{y} .
$$

On the other hand, the energy-momentum in the exterior region (and the corresponding definition of the electric current) can be rewritten in Cartesian coordinates as

$$
\begin{aligned}
& T_{e m}^{t t}=T_{e m}^{z z}=\frac{I^{2}}{2 \pi r}, \\
& T_{e m}^{k j}=-\frac{I^{2}}{2 \pi r^{4}}\left(2 x^{k} x^{j}-r^{2} \delta_{k j}\right) .
\end{aligned}
$$

Therefore, we have

$$
\begin{aligned}
& T_{(0)}^{t t}=U \delta(x) \delta(y)+\frac{I^{2}}{4 \pi} \nabla^{2}\left[\ln \left(\frac{r}{r_{0}}\right)\right]^{2}, \\
& T_{(0)}^{z z}=-T \delta(x) \delta(y)+\frac{I^{2}}{4 \pi} \nabla^{2}\left[\ln \left(\frac{r}{r_{0}}\right)\right]^{2}, \\
& T_{(0)}^{k j}=-I^{2}\left[\delta^{k j} \delta(x) \delta(y)-\frac{\partial_{k} \partial_{j} \ln \left(r / r_{0}\right)}{2 \pi}\right] .
\end{aligned}
$$

\footnotetext{
${ }^{2}$ The calculations are straightforward but lengthy. We will skip the details here and provide the results directly. For the details of these calculations, we refer the reader to Refs. $[7,8]$.
}

Solving the linearized Einstein equations, we obtain

$$
\begin{aligned}
\nabla^{2} h_{t t} & =-4 G_{0}\left\{I^{2} \nabla^{2}\left[\ln \left(\frac{r}{r_{0}}\right)\right]^{2}+2 \pi\left(U-T-I^{2}\right) \delta(x) \delta(y)\right\} \\
& \Rightarrow h_{t t}=-4 G_{0}\left\{I^{2}\left[\ln \left(\frac{r}{r_{0}}\right)\right]^{2}+\left(U-T-I^{2}\right) \ln \left(\frac{r}{r_{0}}\right)\right\},
\end{aligned}
$$

$$
\begin{gathered}
\nabla^{2} h_{z z}=-4 G_{0}\left\{I^{2} \nabla^{2}\left[\ln \left(\frac{r}{r_{0}}\right)\right]^{2}+2 \pi\left(U-T+I^{2}\right) \delta(x) \delta(y)\right\} \\
\Rightarrow h_{z z}=-4 G_{0}\left\{I^{2}\left[\ln \left(\frac{r}{r_{0}}\right)\right]^{2}+\left(U-T+I^{2}\right) \ln \left(\frac{r}{r_{0}}\right)\right\} \\
\nabla^{2} h_{k j}=-4 G_{0}\left[+2 I^{2} \partial_{k} \partial_{j} \ln \left(\frac{r}{r_{0}}\right)\right. \\
\left.+2 \pi \delta_{k j}\left(U-T-I^{2}\right) \delta(x) \delta(y)\right] \\
\Rightarrow h_{k j}=2 G_{0} I^{2} r^{2} \partial_{k} \partial_{j} \ln \left(\frac{r}{r_{0}}\right) \\
-4 G_{0} \delta_{k j}\left(U+T-I^{2}\right) \ln \left(\frac{r}{r_{0}}\right)
\end{gathered}
$$

In order to match the internal and external solutions and obtain the parameters $m, B, n$, and $D$, we need to return to the cylindrical coordinate system:

$$
\begin{aligned}
& g_{t t}=-\left(1+4 G_{0}\left\{I^{2}\left[\ln \left(\frac{r}{r_{0}}\right)\right]^{2}+\left(U-T-I^{2}\right) \ln \left(\frac{r}{r_{0}}\right)\right\}\right) \\
& g_{z z}=1-4 G_{0}\left\{I^{2}\left[\ln \left(\frac{r}{r_{0}}\right)\right]^{2}+\left(U-T+I^{2}\right) \ln \left(\frac{r}{r_{0}}\right)\right\} \\
& g_{r r}=1-2 G_{0} I^{2}-4 G_{0}\left(U+T-I^{2}\right) \ln \left(\frac{r}{r_{0}}\right) \\
& g_{\theta \theta}=r^{2}\left[1+2 G_{0} I^{2}-4 G_{0}\left(U+T-I^{2}\right) \ln \left(\frac{r}{r_{0}}\right)\right]
\end{aligned}
$$

Before presenting the final result we note that the metric (29) does not preserve the feature $g_{R R}=g_{z z}$. We need again to make a change of coordinates, to first order in $G_{0}$,

$$
R=r\left\{1+a_{1}-a_{2} \ln \left(\frac{r}{r_{0}}\right)-a_{3}\left[\ln \left(\frac{r}{r_{0}}\right)\right]^{2}\right\},
$$

where $a_{1}=G_{0}\left(4 T-I^{2}\right), \quad a_{2}=4 G_{0} T$, and $a_{3}=-2 G_{0} I^{2}$. Without loss of generality, let us make $R=r$ and expand the parameters in powers of $\ln \left(r / r_{0}\right)$. In doing that, we finally get 


$$
\begin{aligned}
d s^{2}= & \left\{1-4 G_{0}\left[\left(U-T+I^{2}\right) \ln \left(\frac{r}{r_{0}}\right)+I^{2} \ln ^{2}\left(\frac{r}{r_{0}}\right)\right]\right\}\left(d r^{2}+d z^{2}\right)+r^{2}\left(1-8 G_{0}\left(T-\frac{I^{2}}{2}\right)-4 G_{0}\left(U-T-I^{2}\right) \ln \left(\frac{r}{r_{0}}\right)\right. \\
& \left.-4 G_{0} I^{2} \ln ^{2}\left(\frac{r}{r_{0}}\right)\right] d \theta^{2}-\left\{1+4 G_{0}\left[I^{2} \ln ^{2}\left(\frac{r}{r_{0}}\right)+\left(U-T-I^{2}\right) \ln \left(\frac{r}{r_{0}}\right)\right]\right\} d t^{2} .
\end{aligned}
$$

In the next section, we use this metric to compute the deficit angle and the deflection of light rays by a currentcarrying string.

\section{Deficit angle and the deflection of light rays by a current-carrying string}

Now that we have calculated the metric (33) of a timelike current-carrying string, we can compute some quantities associated with it, such as the deficit angle and the deflection of light rays. The deficit angle is calculated with the help of the expression

$$
\delta_{\theta}=2 \pi\left(1-\frac{1}{\sqrt{g_{\rho \rho}}} \frac{d}{d \rho} \sqrt{g_{\theta \theta}}\right) .
$$

For the metric (33) it is, to first order in $G_{0}$,

$$
\delta_{\theta}=4 \pi G_{0}\left(U+T-2 I^{2}\right)+O\left(G_{0}^{2}\right) .
$$

It is clear that, if $I$ is zero (the neutral string case), the deficit angle (34) reduces to $\delta_{\theta}=8 \pi G_{0} U$ which is of order $\sim 10^{-6}$ for grand unified theory (GUT) strings.

The deflection of the light ray is given by the expression

$$
\Delta \theta=2 \int_{\rho_{\min }}^{\infty} d \rho\left(-\frac{g_{\theta \theta}^{2} p^{-2}}{g_{\rho \rho} g_{t t}}-\frac{g_{\theta \theta}}{g_{\rho \rho}}\right)^{-1 / 2}-\pi .
$$

As in [7] we obtain, knowing that $p$ is the impact parameter,

$$
\Delta \theta=4 \pi G_{0}\left\{U-I^{2}\left[\frac{3}{2}-\ln \left(\frac{p}{r_{0}}\right)\right]\right\}+4 \pi \ln (2) I^{2} G_{0}+O\left(G_{0}^{2}\right) .
$$

From expression (36), we can observe some remarkable features of the deflection of light due to a timelike currentcarrying string in the scalar-tensor gravity. First of all, there is no dependence on the tension in the deflection of light rays. This feature is reminiscent of the neutral string. The second feature is the dependence on the impact parameter $p$ due to the presence of the current. This is due to the fact that the inclusion of an electromagnetic field actually increases the total energy with the distance to the string core and, as a consequence, the deflection of light is more intense. Finally, we notice that the dilaton field amplifies this effect since its inclusion also contributes to increasing the total energy of the system.

\section{FORMATION AND EVOLUTION OF THE WAKES AND THE ZEL'DOVICH APPROXIMATION}

A relevant mechanism to understand structure formation by cosmic strings involves long strings moving with relativistic speed in the normal plane, giving rise to velocity perturbations in their wake [2]. Matter through which a long string moves acquires a boost in the direction of the surface swept out by the string. Matter moves toward this surface by gravitational attraction and, as a consequence, a wake is formed behind the string. In what follows, we will study the implications of a timelike current in the formation and evolution of a wake behind the string that generates the metric (33). For this purpose, we will mimic this situation with a simple model consisting of cold dark matter composed of nonrelativistic collisionless particles moving past a long string. In order to make a quantitative description of accretion onto wakes, we use the Zel'dovich approximation, which consists in considering the Newtonian accretion problem in an expanding universe using the method of linear perturbations.

To start with, we first compute the velocity perturbation of massive particles moving past the string. If we consider that the string is moving with normal velocity $v_{s}$ through matter, the velocity perturbation can be calculated with the help of the gravitational force due to the metric (33):

$$
\begin{aligned}
u= & 8 \pi G_{0} U v_{s} \gamma+\frac{\pi G_{0}}{v_{s} \gamma}\left[2 \alpha\left(\phi_{0}\right)^{2}\left(U+T+I^{2}\right)-\left(U-T-I^{2}\right)\right. \\
& \left.-2 \ln \left(\frac{r}{r_{0}}\right) I^{2}\right]
\end{aligned}
$$

with $\gamma=\left(1-v_{s}^{2}\right)^{-1 / 2}$. The first term in Eq. (37) is equivalent to the relative velocity of particles flowing past a string in general relativity. The other terms come as a consequence of the scalar-tensor coupling of the gravitational interaction and the superconducting properties of the string.

Let us suppose now that the wake was formed at $t_{i}$ $>t_{e q}$. The physical trajectory of a dark particle can be written as

$$
h(\vec{x}, t)=a(t)[\vec{x}+\psi(\vec{x}, t)]
$$

where $\vec{x}$ is the unperturbed comoving position of the particle and $\psi(\vec{x}, t)$ is the comoving displacement developed as a consequence of the gravitational attraction induced by the wake on the particle. Suppose, for simplification, that the wake is perpendicular to the $x$ axis [assuming that $d z=0$ in the metric (33) and $r=\sqrt{x^{2}+y^{2}}$ ] in such a way that the only 
nonvanishing component of $\psi$ is $\psi_{x}$. Therefore, the equation of motion for a dark particle in the Newtonian limit is

$$
\ddot{h}=-\nabla_{h} \Phi,
$$

where the Newtonian potential $\Phi$ satisfies the Poisson equation

$$
\nabla_{h}^{2} \Phi=4 \pi G_{0} \rho,
$$

where $\rho(t)$ is the dark matter density in a cold dark matter universe. For a flat universe in the matter-dominated era, $a(t) \sim t^{2 / 3}$. Therefore, the linearized equation for $\psi_{x}$ is

$$
\ddot{\psi}+\frac{4}{3 t} \dot{\psi}-\frac{2}{3 t^{2}} \psi=0
$$

with appropriate initial conditions: $\psi\left(t_{i}\right)=0$ and $\dot{\psi}\left(t_{i}\right)=$ $-u_{i}$. Equation (41) is the Euler equation whose solution is easily found:

$$
\psi(x, t)=\frac{3}{5}\left[\frac{u_{i} t_{i}^{2}}{t}-u_{i} t_{i}\left(\frac{t}{t_{i}}\right)^{2 / 3}\right] .
$$

Calculating the comoving coordinate $x(t)$ using the fact that $\dot{h}=0$ in the "turnaround," 3 we get

$$
x(t)=-\frac{6}{5}\left[\frac{u_{i} t_{i}^{2}}{t}-u_{i} t_{i}\left(\frac{t}{t_{i}}\right)^{2 / 3}\right] .
$$

With the help of Eq. (42) we can compute both the thickness $d(t)$ and the surface density $\sigma(t)$ of the wake [1]. We have, then, respectively (to first order in $G_{0}$ ),

$$
\begin{aligned}
d(t) \approx & \frac{12}{5}\left(\frac{t}{t_{i}}\right)^{1 / 3}\left\{8 \pi G_{0} U v_{s} \gamma+\frac{\pi G_{0}}{v_{s} \gamma}\left[2 \alpha\left(\phi_{0}\right)^{2}\left(U+T+I^{2}\right)\right.\right. \\
& \left.\left.-\left(U-T-I^{2}\right)-2 \ln \left(\frac{r}{r_{0}}\right) I^{2}\right]\right\} \\
\sigma(t) \approx & \frac{2}{5} \frac{1}{v_{s} \gamma t}\left(\frac{t}{t_{i}}\right)^{1 / 3}\left[8 U\left(v_{s} \gamma\right)^{2}+2 \alpha\left(\phi_{0}\right)^{2}\left(U+T+I^{2}\right)\right. \\
& \left.-\left(U-T-I^{2}\right)-2 \ln \left(\frac{r}{r_{0}}\right) I^{2}\right],
\end{aligned}
$$

\footnotetext{
${ }^{3}$ The moment when the dark particle stops expanding with the Hubble flow and starts to collapse onto the wake.
}

where we have used the fact that $\rho(t)=1 / 6 \pi G_{0} t^{2}$ for a flat universe in the matter-dominated era and that the wake was formed at $t_{i} \sim t_{e q}$. Clearly, from Eq. (43) we see that the presence of the current causes the accretion mechanism by wakes to diverge.

\section{CONCLUSION}

Inclusion of a current in the internal structure of a cosmic string could drastically change the predictions of such models in the microwave background anisotropies. In particular, a current of a timelike type could bring some divergences, leading to some unbounded gravitational effects [9]. In this work we studied the effects of a timelike current string in the mechanism of wake formation. For this purpose, we first studied the gravitational properties of the spacetime generated by this string in the framework of scalar-tensor gravities. We analyzed the dependence of the deficit angle and the deflection of light on the current in this spacetime. Then we carried out an investigation of the mechanism of formation and evolution of wakes in this framework, showing the explicit contribution of the current to this effect.

Wakes produced by moving strings can provide an explanation for filamentary and sheetlike structures observed in the universe. A wake produced by the string in one Hubble time has the shape of a strip of width $\sim v_{s} t_{i}$. With the help of the surface density (43) we can compute the wake's linear mass density, say $\tilde{\mu}$,

$$
\begin{aligned}
\sigma(t) \approx & \frac{2}{5} \frac{1}{\gamma t}\left(\frac{t}{t_{i}}\right)^{1 / 3}\left[8 U\left(v_{s} \gamma\right)^{2}+2 \alpha\left(\phi_{0}\right)^{2}\left(U+T+I^{2}\right)\right. \\
& \left.-\left(U-T-I^{2}\right)-2 \ln \left(\frac{r}{r_{0}}\right) I^{2}\right] .
\end{aligned}
$$

If the string moves more slowly or if we extrapolate our results to earlier epochs, we see that the logarithmic term will cause divergences and the mechanism of forming wakes will break down.

\section{ACKNOWLEDGMENTS}

The authors would like to thank CAPES for partial financial support in the context of the PROCAD program. A.L.N.O. would like to thank CAPES for a Ph.D. grant. M.E.X.G. is on leave from Depto. de Matemática, Universidade de Brasília.
[1] A. Vilenkin and E.P.S. Shellard, Cosmic Strings and Other Topological Defects (Cambridge University Press, Cambridge, England, 1994).

[2] J. Silk and A. Vilenkin, Phys. Rev. Lett. 53, 1700 (1984).

[3] T. Vachaspati, Phys. Rev. Lett. 57, 1655 (1986); A. Stebbins, S. Veeraraghavan, R.H. Brandenberger, J. Silk, and N. Turok, As- trophys. J. 1, 322 (1987); N. Deruelle and B. Linet, Class. Quantum Grav. 5, 55 (1988); W. Hiscock and B. Lail, Phys. Rev. D 37, 869 (1988); L. Perivolaropoulos, R.H. Brandenberger, and A. Stebbins, ibid. 41, 1764 (1990).

[4] T. Vachaspati, Phys. Rev. D 45, 3487 (1992).

[5] S.R.M. Masalskiene and M.E.X. Guimarães, Class. Quantum 
Grav. 17, 3055 (2000).

[6] V.B. Bezerra and C.N. Ferreira, Phys. Rev. D 65, 084030 (2002).

[7] P. Peter and D. Puy, Phys. Rev. D 48, 5546 (1993).

[8] C.N. Ferreira, M.E.X. Guimarães, and J.A. Helayel-Neto, Nucl. Phys. B581, 165 (2000).

[9] P. Peter, Class. Quantum Grav. 11, 131 (1994).

[10] B. Carter, P. Peter, and A. Gangui, Phys. Rev. D 55, 4647 (1997).

[11] V.C. Andrade, P. Peter, and M.E.X. Guimarães, gr-qc/0101039.
[12] H.B. Nielsen and P. Olesen, Nucl. Phys. B61, 45 (1973).

[13] E. Witten, Nucl. Phys. B249, 557 (1985).

[14] B. Carter, in Formation and Evolution of Cosmic Strings, edited by G.W. Gibbons, S.W. Hawking, and T. Vachaspati (Cambridge University Press, Cambridge, England, 1990).

[15] L. Witten, in Gravitation, edited by L. Witten (Wiley, New York, 1962).

[16] M.A. Melvin, Phys. Lett. 8, 64 (1964).

[17] B. Linet, Class. Quantum Grav. 6, 435 (1989). 